\title{
Several Different Remote Sensing Image Classification Technology Analysis
}

\author{
Xiangwei Liu \\ Foundation Department, PLA University of Foreign Languages, Luoyang 471003, China \\ E-mail: liuxwletter@163.com
}

Received: July 10, 2011

Accepted: September 2, 2011

doi:10.5539/jsd.v4n5p167

\begin{abstract}
Remote sensing (RS) image classification is an important content of RS research area. How to solve the classification of the multi-category image, avoid the existence of "same objects with different spectrums" and "different spectrums with same objects" phenomemon, and achieve certain accuracy requirement is a key question in RS application research, which has the very important practical significance especially in the military uffairs scout, geological survey and civil fields. By using the LANDSAT-7 ETM + RS image with the support of the project which investigated geology and mineral resources in the around 1:50 thousand regional Sepikou, Mulei County of Xinjiang in 2008, the improved BP neural network model is designed in this paper by the adaptive learning rate and the additional momentum, and compared with the traditional classification methods. Through the precision analysis by the error matrix, the result shows that the total classification accuracy which uses improved BP NN classification of RS image is $89.8 \%$, and the classification accuracy of RS image is improved obviously, and is a kind of effective classification method.
\end{abstract}

Keywords: RS image classification, Improved BP NN, Traditional classification, Precision analysis

\section{Introduction}

RS technology is integrated as an American scholar in 1960 put forward.(Chen, 1990, p.10-19).Since the 90s, rapid development of space RS technology, application field continued to expand and deepen ceaselessly, RS technology in geological survey, mineral exploration, geological evaluation, geological disaster monitoring and basic geological research has played an increasingly large role.(Chang,2004, p. 206-210). The paper is used the ETM RS images, combined with the ERDAS Imagine 9.1 RS image processing software, pre-processing of RS images, aided by map information and the field test data,using the adaptive learning rate and the additional momentum method on the traditional BP NN improved, with the MATLAB NN toolbox and achieves improved BP NN classification of RS images in the research area, and compares with other classification methods, finds similar geographic region or geological features similar to the process of rapid classification,to improve the level of information. The technical route is shown in Figure 1.

\section{The research area and $\mathrm{RS}$ data range}

\subsection{The research area}

The research area is located in Mulei County east of Hui Autonomous prefecture, Changji,Xinjiang and Qijiaojing twon of Hami district. Geographic coordinates: east longitude $91^{\circ} 00^{\prime}$ to $91^{\circ} 30$ '; north latitude $43^{\circ}$ $30^{\prime}$ to $43^{\circ} 50^{\prime}$. Administrative districts belongs to the Mulei County, Hami city jurisdiction shown in Figure 2.

\subsection{RS data range}

The paper uses the LANDSAT-7 ETM + RS images as the main data source, it is the land of the gain of the no. 7 satellite RS data. The LANDSAT-7 ETM + has 8 bands, which TM1, 2, 3 band for visible bands, TM4 for the near-infrared bands, TM5, 7 for the mid-infrared bands, the resolution are $30 \mathrm{~m}$, TM6 for thermal infrared bands for $60 \mathrm{~m}$, TM8 for the additional panchromatic resolution of up to $15 \mathrm{~m}$. RS image classification is usually used in addition to the sixth band other than the 6 band images, which is TM1, TM2, TM3, TM4, TM5, TM7. By reading the six TM image gray value of each pixel, you can construct a sample of six-dimensional input sample space: $\mathrm{Vi}=\{\mathrm{Vil}, \mathrm{Vi2}, \mathrm{Vi} 3, \mathrm{Vi4}, \mathrm{Vi5}, \mathrm{Vi} 6\}$.

\section{RS image preprocessing}

The research area is $512 \times 512$ size of RS image from the original image, using the query box method(Dang, 2003, p.1-6) with ERDAS IMAGINE9.1 software, as shown in Figure 3. RS image after geometric correction, 
radiation correction, band ETM741 of false color synthesis, and full-color band 8 fusion to improve resolution(Tang, 2004, p.170-176), to obtain new RGB color synthesis image as shown in Figure 4.

\section{Extract data}

By understanding the actual situation in the region and the visual interpretation of the image, the region can be divided into five categories. Start the ERDAS IMAGINE9.1 software, open the classified image, according to various features in the reflectance in different bands is different for each surface features selects 200 samples, total sample size of 1000 , of which 500 as a training sample, 500 as test samples.

\section{5. improved BP NN model}

\subsection{Building a model}

The improved BP NN model is based on the error back-propagation learning algorithm for multilayer perceptron model structure (BPNN). The paper determines the improved BP model parameters are: three layer structure of BP network; 2, 3, 5, 6, four bands of data, that the number of neurons in input layer is 4 , the input vector of pretreatment, data limitations in $[0,1]$; output nodes with the same number of classification categories, namely, the output node is 5 . After many experiments, the node amount in the concealed layer is 22 , initial weights are initialized to $[-1,1]$ between the random number, momentum factor is set to 0.001 , expected error is 0.01 , using the Sigmoid function and the hyperbolic tangent function, establishes the BP NN structure.

\section{2 data simulation}

In the MATLAB NN toolbox, there are very easy function to build neural network.

The order is “net = newff(minmax(P),[22,5], \{'tansig','logsig'\},'traingdx','learngdm','mse');

This order establishes the network object and initializes the network weight and deflection, and its input is matrix of $4 \times 500$, the transfer function of the first layer is "tansig", the transfer function of the output layer is "logsig", and the training function is "traingdx". Using train function simulation, when training to 558 times, the network reaches a predetermined classification speed and error rate.

In order to test the improved BP model is correct, chooses the same test data to test, testing by the improved BP model output and the desired output error is very small, the forecast result and the actual situation is almost exactly the same. The paper sums up the best network training parameters: the initial weight of 0.1 , learning rate of 0.9 , increasing rate of learning speed of 1 , decreasing rate of learning speed of 0.05 , momentum factor of 0.001 , the global network error of 0.01.It is obvious that it is feasible that to adopt the improved BP NN to classify the RS images on the platform of the MATLAB NN toolbox.

\subsection{Improved BP model classification in the research area}

Through the testing of the data, the improved BP NN accord with the requirements, and then the program reads the data of the whole image, and classify it. The classification image is seen in Figure 5.

\section{Traditional RS image classification}

\subsection{Supervised classification}

The supervised classification(Dang, 2003, p.1-6)is from the research area selected region of interest as training samples. According to the known interested region is provided in the training sample, by selecting the characteristic parameters of training samples, and establishs corresponding difference function to the training sample pixel classification. The supervised classification method mainly adopts maximum likelihood classification and minimum distance classification algorithm.

The maximum likelihood(Tong, 2006, p.113-118)is calculated point by point in each image pixel data associated with each category of a given degree of likelihood, and points the pixel to the likelihood of the largest categories of methods. The classification image is seen in Figure 6.

The minimum distance method is based on the feature space distance as the basis of pixel classification(Dang, 2003, p.1-6), Including the minimum distance discriminant method and nearest neighbor classification. Minimum distance classification is simple in principle, classification accuracy is not high, but has the fast calculation speed, it can be used in the quick scan classification(Zhang, 2005, p.102-105). The classification image is seen in Figure 7.

\subsection{Unsupervised Classification}

Need not be known samples and classification on computer supervision and training classifiers, but according to the image data characteristics, namely, these data represent the spectrum characteristics of the similarity and 
dissimilarity to classification, called the unsupervised classification(Liu, 2007, p.156-159). In the supervision and classification, the ISDATA algorithm is compared commonly used, that is, the iterative self-organizing data analysis technique. The classification image is seen in Figure 8.

\section{Classification result analysis}

Any image classification process will have error, resulting in the classification errors are a variety of reasons. Classification error in an image is not randomly distributed, but show certain systematic and regularity. The error matrix (i.e. the confusion matrix) (Dang, 2003, p.1-6)is usually used to denote the precision of the classification result in the RS image classification, and based on that, various statistics could be computed and the statistical test could be implemented, and the classification precisions based the total and various ground types could be obtained finally. Generally choose the overall accuracy and Kappa values to evaluate the training samples.

The precision evaluation error matrix should be established, the precisions of the improved BP NN classification, Maximum likelihood classification, Minimum distance classification and Unsupervised classification are respectively evaluated, and the results are shown in Table 1.

Through the analysis of table 1 can be found that the improved BP NN classification than the maximum likelihood classification method, the minimum distance method, unsupervised classification has a certain degree of improvement.

\section{Conclusions}

Taking these results ,the traditional classification method because of their conditions, has been unable to meet the requirement of practical application. The total precision of the improved BP NN classification method is $89.8 \%$, it is a kind of effective classification method, and it can improve the classification accuracy. The improved BP NN can well simulate the nonlinear system, it has strong self-organizing, adaptation and learning ability, and using the advantage, it could be also extended in the other resources investigation.

\section{References}

Chen, Shupeng. (1990). RS dictionary. (3rd ed.). Beijing: Science Press, (Chapter 1).

Chang, Qingrui, Jiang, Pingan \&Zhou ,yong. (2004). Introduction of RS technology. (3rd ed.).Beijing: Science Press, (Chapter 6).

Dang, Anrong, Wang, Xiaodong \& Chen, Xiaofeng. (2003). ERDAS Imagine Remote Sensing Image Processing Method. (3rd ed.). Beijing: Tsinghua University Press, (Chapter 1).

Liu,Yuming. (2007). Based on the Improved BP NN of PID Control Method Research. Graduate School of Chinese Academy of Sciences, 160,156-159.

Tang, Guoan, Zhang, Youshun \& Liu, Yongmei. (2004). RS Digital Image Processing. (3rd ed.). Beijing: Science Press, (Chapter 4).

Tong, Qingxi, Zhang, Ping \& Zheng, Lanfen. (2006). Hyperspectral RS of Multi-Disciplinary Applications. (3rd ed.).Beijing: Electronics Industry Press.May, (Chapter 3).

Zhang, Liangpei \& Zhang,Lifu. (2005). Hyperspectral RS. (3rd ed.).Huibei: Wuhan University Press. October, (Chapter 3).

Table 1. Comparison of different classification

\begin{tabular}{|l|c|c|c|c|}
\hline & Improved BP NN & Maximum likelihood & Minimum distance & $\begin{array}{l}\text { Unsupervised } \\
\text { classification }\end{array}$ \\
\hline $\begin{array}{l}\text { Total } \\
\text { precision }\end{array}$ & $89.8 \%$ & $80.3 \%$ & $75.8 \%$ & $66.7 \%$ \\
\hline Khat & $86.4 \%$ & $78.2 \%$ & $70.5 \%$ & $59.8 \%$ \\
\hline
\end{tabular}




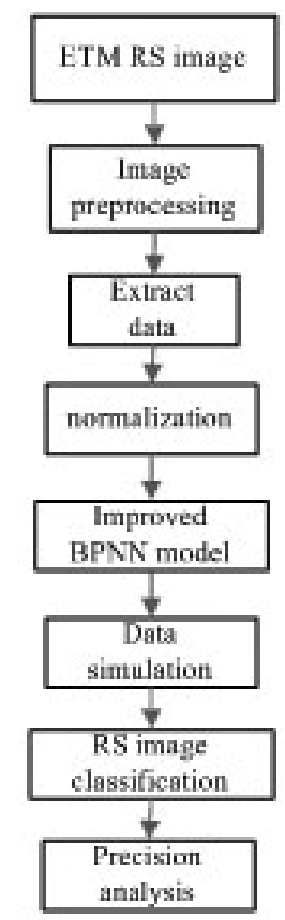

Figure 1. Technology Roadmap

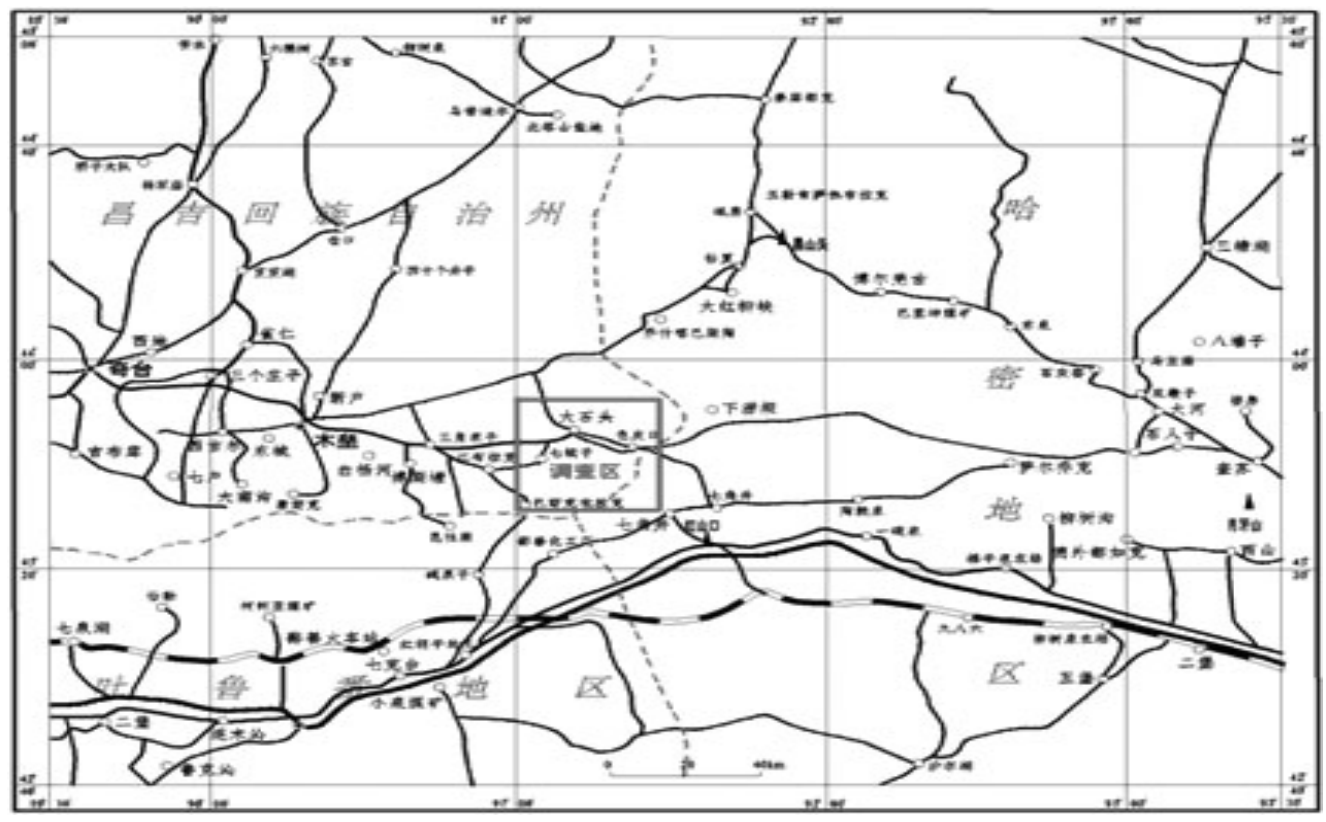

Figure 2. Research Area Map 


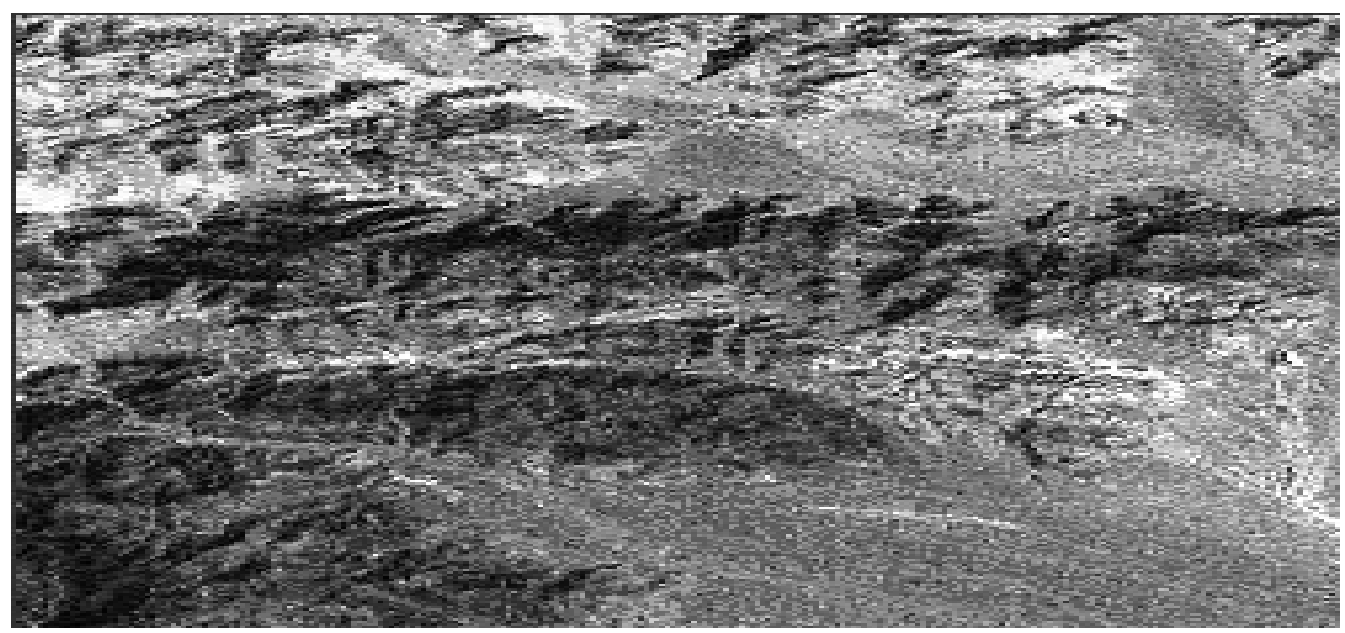

Figure 3. Remote Sensing Image

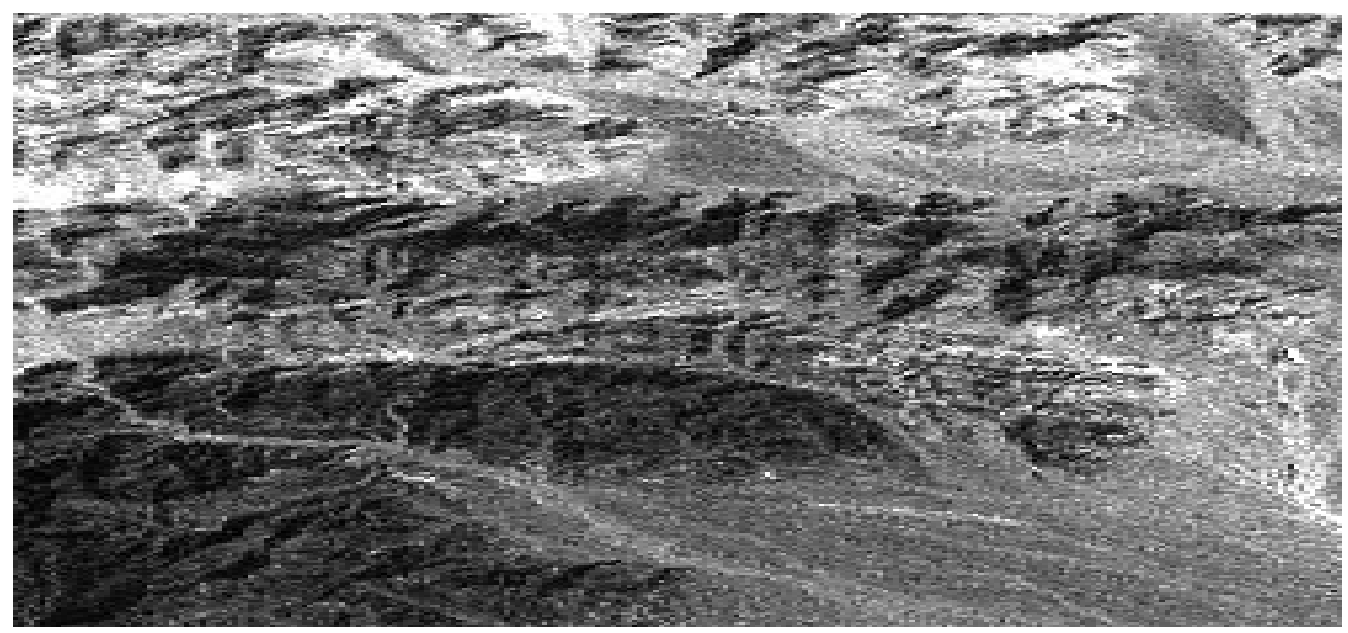

Figure 4. Fusion Image

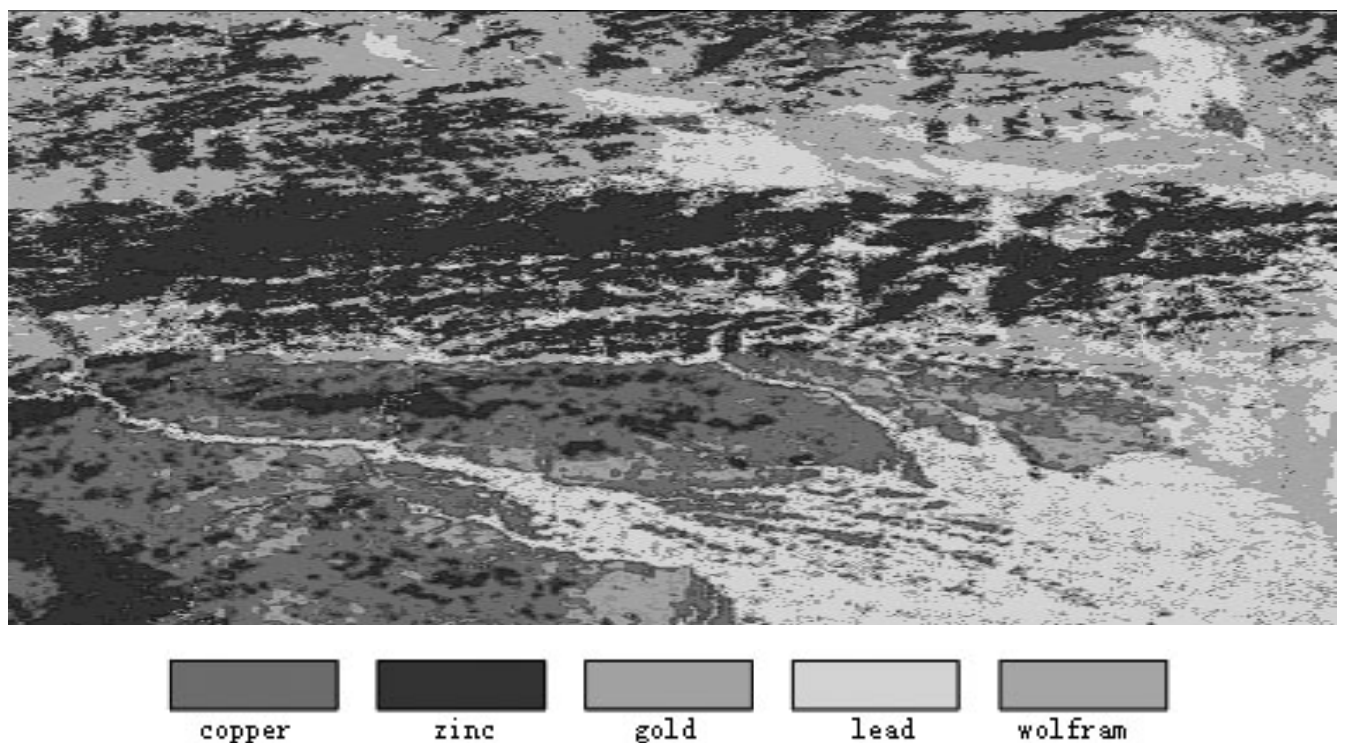

Figure 5. Remote Sensing Image Classification Based on the Improved BP NN 


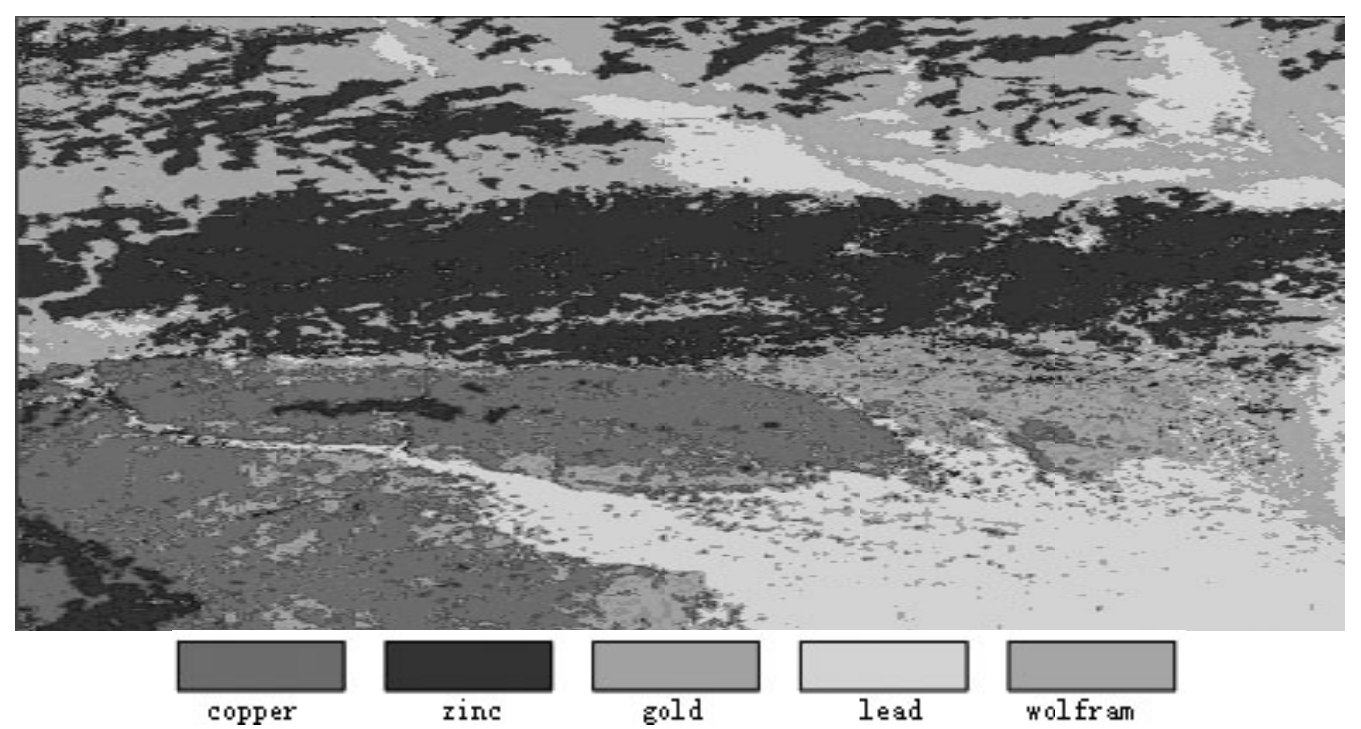

Figure 6. Remote Sensing Image Classification Based on the Maximum Likelihood

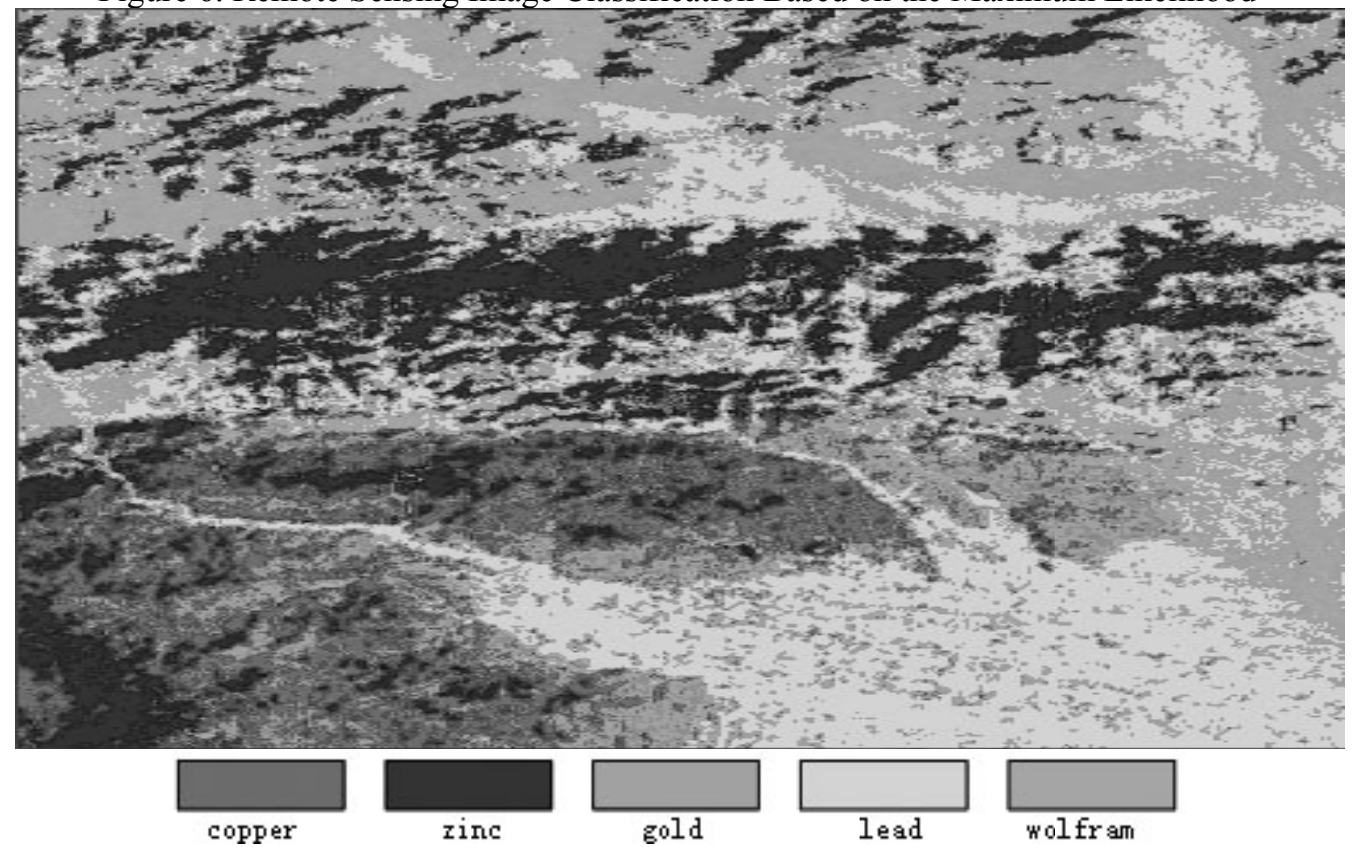

Figure 7. Remote Sensing Image Classification Based on the Minimum Distance 


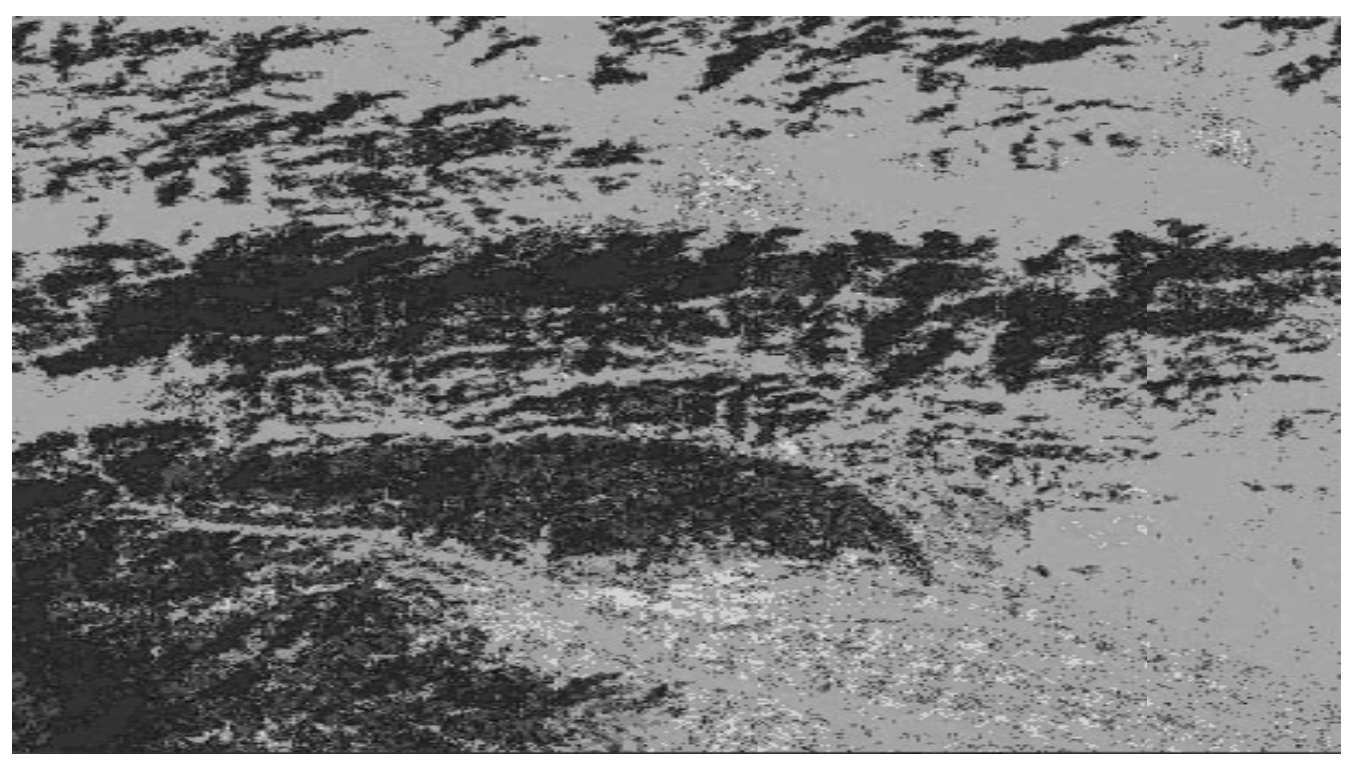

Figure 8. Remote Sensing Image Classification Based on the Unsupervised Classification 